\title{
多种重离子辐照对嗍硅酸盐玻璃机械性能的影响
}

\author{
张晓阳，彭海波，刘枫飞，赵彦，孙梦利，管明， \\ 张冰㫪，杜 金金，袁伟，王铁山 \\ (兰州大学 核科学与技术学院, 兰州 730000)
}

摘 要: 研究辐照导致嗍硅酸盐玻璃机械性能的影响, 对高放废物的长期安全处置具有重要的意义。本工作采用 $0.3 \mathrm{MeV}$ 的 $\mathrm{P}$ 离子、 $4 \mathrm{MeV}$ 的 $\mathrm{Kr}$ 离子、 $5 \mathrm{MeV}$ 的 $\mathrm{Xe}$ 离子以及 $8 \mathrm{MeV}$ 的 $\mathrm{Au}$ 离子分别辐照嗍硅酸盐玻璃, 利用纳 米压痕技术表征了辐照前后样品的硬度和模量。结果表明: 嗍硅酸盐玻璃的硬度和模量在一定范围内会随着辐照剂 量的增大而减小，辐照达到 $0.1 \mathrm{dpa}$ 时硬度和模量变化趋于饱和，此时硬度下降了 $35 \%$, 模量下降了 $18 \%$; 而且不同 种类的离子辐照对硼硅酸盐玻璃的硬度和模量造成的变化趋势基本相同。使用掠入射 $\mathrm{X}$ 射线衍射仪对样品晶态结 构进行了分析, 发现辐照后硼硅酸盐玻璃仍保持非晶状态。利用 Raman 光谱对辐照后样品的微观结构的变化进行 了表征, 发现辐照会导致玻璃网络结构发生改变, 玻璃的聚合度下降, 无序度增加。本工作还证明了离子辐照导致 玻璃机械性能变化的主要因素是离子在样品中的核能量沉积导致玻璃结构的改变。

关 键 词: 硼硅酸盐玻璃; 辐照效应; 机械性能

中图分类号: TQ171 文献标识码: A

\section{Mechanical Properties of Borosilicate Glass with Different Irradiation of Heavy Ions}

\author{
ZHANG Xiao-Yang, PENG Hai-Bo, LIU Feng-Fei, ZHAO Yan, SUN Meng-Li, \\ GUAN Ming, ZHANG Bing-Tao, DU Xin, YUAN Wei, WANG Tie-Shan
}

(School of Nuclear Science and Technology, Lanzhou University, Lanzhou 730000, China)

\begin{abstract}
To understand the evolutions in mechanical properties of borosilicate glass under irradiation is significant for evaluating its performance after long-term interaction with irradiation environment. In this study, borosilicate glass samples were irradiated with $0.3 \mathrm{MeV}$ P-ions, $4 \mathrm{MeV}$ Kr-ions, $5 \mathrm{MeV}$ Xe-ions and $8 \mathrm{MeV}$ Au-ions, respectively. Modification of the samples' mechanical properties before and after radiation was investigated with nano-indentation measurement. With increase of irradiation dose, both hardness and modulus of samples irradiated with $\mathrm{P}, \mathrm{Kr}, \mathrm{Xe}$ and $\mathrm{Au}$ ions drop and then saturate at $0.1 \mathrm{dpa}$. The trends of hardness variations for the sample irradiated with four kinds of ions at different energies are similar, and the change of modulus also follows the same rule. The maximum variation of NBS2 sample' hardness and modulus are about 35\% and 18\%, respectively. The grazing incident X-ray diffraction (GIXRD) was employed to analyze the phase structure. The results indicate that borosilicate glass still remains amorphous after irradiation. The microstructure evolutions of irradiated borosilicate glasses were characterized by Raman spectroscopy. The decrease of network polymerization and the increase of structural disorder are suggested by Raman spectra. In addition, the results also suggest that the main factor for the evolutions of the hardness and modulus of the
\end{abstract}

收稿日期：2018-09-18; 收到修改稿日期：2018-11-15

基金项目：国家自然科学基金(11505085, U1867207); 中央高校基本科研业务费(lzujbky-2018-72, lzujbky-2018-74); 甘肃省 引导科技创新发展专项资金(2018ZX-07)

National Natural Science Foundation of China(11505085, U1867207); Fundamental Research Funds for the Central Universities (lzujbky-2018-72, lzujbky-2018-74); DSTI Foundation of Gansu(2018ZX-07)

作者简介: 张晓阳(1994-), 男, 硕士研究生. E-mail: 1843558514@qq.com

通讯作者: 彭海波，副教授.E-mail: penghb@1zu.edu.cn; 王铁山，教授.E-mail: tswang@1zu.edu.cn 
borosilicate glass under ion irradiation is the change of the deformation of glass network, which was caused by nuclear energy deposition in samples.

Key words: borosilicate glass; radiation effect; mechanical properties

随着核能在人类社会中的广泛应用，核能利用中 的一些问题也逐渐凸显。核能利用过程中产生的放射性 废物处理问题长期困扰着人类。尤其是高放废物, 它 的放射性比活度高, 释热率高, 含有一些半衰期长、 生物毒性大的核素, 因此需要将其永久隔离。为避免 高放废物影响到人类居住环境, 国际上(包括我国在 内)通常的做法是“玻璃固化-地质处置” 硼硅酸盐玻 璃是当前国际上主要使用的高放废物固化材料。然而 人们对在长期的地质处置中, 作为高放废物包容体的 玻璃在辐照条件下的性能变化仍然了解有限。因此, 人们对玻璃固化体的辐照效应做了大量的研究 ${ }^{[1-3]}$ 。

Peuget 等 ${ }^{[4-5]}$ 使用不同种类的离子辐照玻璃来模 拟辐照条件下玻璃性质的改变, 并且指出离子辐照后 玻璃固化体的硬度会下降。Weber 等 ${ }^{[6]}$ 研究了 ${ }^{238} \mathrm{Pu}$ 和 ${ }^{244} \mathrm{Cm}$ 掺杂模拟高放废物玻璃固化体机械性能随辐 照剂量的变化, 发现玻璃固化体硬度、模量均随辐照 剂量的增加而下降。Abbas 等 ${ }^{[7]}$ 采用 $2.1 \mathrm{MeV} \mathrm{Kr}$ 离子 在室温下辐照的方式, 研究发现 $\mathrm{Kr}$ 离子辐照后硼 硅酸盐玻璃硬度下降了约 $20 \%$ 。Ollier 等 ${ }^{[8]}$ 研究了经 $\beta$ 辐照后的玻璃固化体的拉曼光谱, 发现混合碱效 应可以减弱辐照后玻璃结构的变化。Wang 等 ${ }^{[9-10]}$ 对辐照后嗍硅酸盐玻璃进行了纳米硬度测试, 发现 硬度和模量都有不同程度的下降; 同时通过分析经 重离子辐照后玻璃的拉曼谱证实了会产生分子氧。 Luo 等 ${ }^{[11]}$ 对不同组分玻璃固化体的浸出行为进行了 研究。Delaye 等 ${ }^{[12]}$ 使用分子动力学方法模拟了经离 子辐照后嗍硅酸盐玻璃硬度等性能的变化, 与实验 结果相符。辐照导致硼硅酸盐玻璃机械性能的变化 已经做了大量研究。然而, 对于嗍硅酸盐玻璃的辐 照损伤机理尚未完全解释清楚, 如辐照对玻璃硬度 和模量的具体影响。因此, 在这些方面仍需要更多 的实验和理论研究。

本工作分别采用 $0.3 \mathrm{MeV}$ 的 $\mathrm{P}$ 离子、 $4 \mathrm{MeV}$ 的 $\mathrm{Kr}$ 离子、 $5 \mathrm{MeV}$ 的 $\mathrm{Xe}$ 离子以及 $8 \mathrm{MeV}$ 的 $\mathrm{Au}$ 离子 辐照嗍硅酸盐玻璃, 并对辐照前后的样品进行纳米 压痕测试, 以探究嗍硅酸盐玻璃经过四种种类和能 量各不相同的离子辐照后, 其硬度和模量的变化。

\section{1 实验方法}

\section{1 辐照实验}

实验使用的嗍硅酸盐玻璃(NBS2)组分质量百
分比 $\mathrm{SiO}_{2}(65.2 \%) 、 \mathrm{~B}_{2} \mathrm{O}_{3}(18.8 \%)$ 和 $\mathrm{Na}_{2} \mathrm{O}(16.0 \%)$, 其 中 $\mathrm{Na}_{2} \mathrm{O}$ 组分在实际炼制过程中用碳酸钠代替。玻 璃样品是由北京特种玻璃研究院炼制, 炼制过程是 先将原料升温至 $800{ }^{\circ} \mathrm{C}$ 去碳酸化, 然后升温至 $1200{ }^{\circ} \mathrm{C}$ 使原料充分反应，最后降至 $500{ }^{\circ} \mathrm{C}$ 恒温 $24 \mathrm{~h}$ 退火, 以去除样品中的残余应力。最后将炼制好的 玻璃切割成 $10 \mathrm{~mm} \times 10 \mathrm{~mm} \times 1 \mathrm{~mm}$ 的方块, 并进行抛 光就得到了实验所需的样品。

在北京师范大学 $\mathrm{HVE}-400 \mathrm{kV}$ 离子注入机串 列加速器上完成 $0.3 \mathrm{MeV} \mathrm{P}$ 离子辐照嗍硅酸盐玻璃 的实验。在兰州中国近代物理研究所 $320 \mathrm{kV}$ 高压平 台上开展 $4 \mathrm{MeV}$ 的 $\mathrm{Kr}$ 离子和 $5 \mathrm{MeV}$ 的 $\mathrm{Xe}$ 离子辐 照硼硅酸盐玻璃实验。在四川大学的 $3 \mathrm{MV}$ 串列静 电加速器上完成 $8 \mathrm{MeV}$ 的 $\mathrm{Au}$ 离子的辐照实验。辐 照是在真空靶室中进行, 辐照样品时环境温度为室 温, 靶室真空度高于 $1 \times 10^{-6} \mathrm{~Pa}$ 。用四种离子分别辐 照嗍硅酸盐玻璃, 通过 SRIM 模拟其损伤分布, 如 图 1 所示。辐照剂量如表 1 所示。

\section{2 测试方法}

使用苏州纳米研究所的 Nano Indenter G200 型

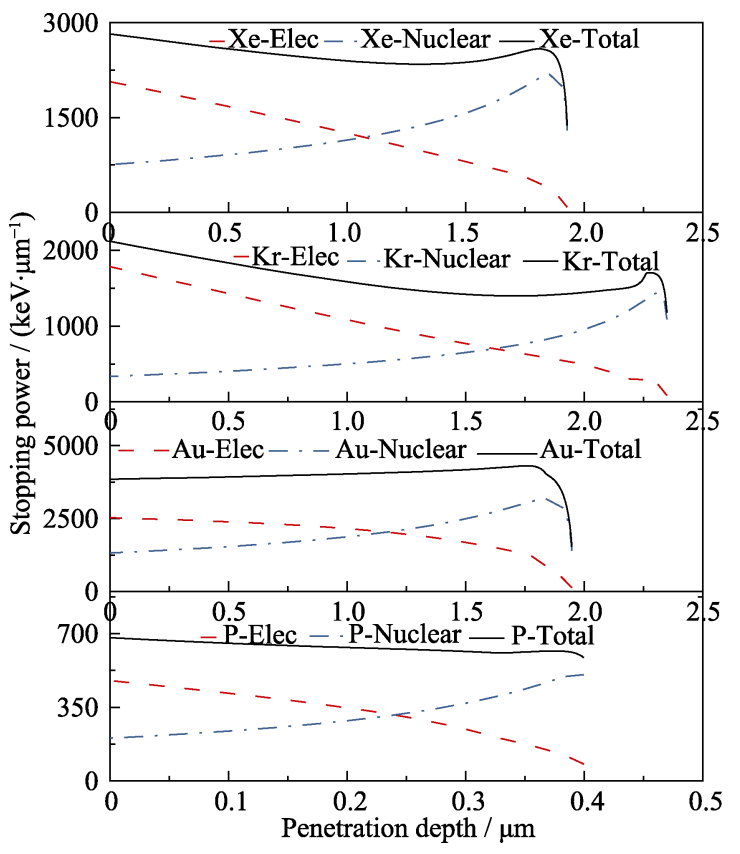

图 1 重离子辐照硼硅酸盐玻璃电子能损、核能损以及总能 损分布曲线

Fig. 1 Electronic energy loss, nuclear energy loss and total energy loss distribution curves of borosilicate glass with irradiation of heavy ions 
表 1 重离子辐照硼硅酸盐玻璃剂量表

Table 1 Doses of heavy ions for the irradiation

\begin{tabular}{|c|c|c|c|c|c|c|}
\hline Ion & Energy/MeV & Range/ $\mu \mathrm{m}$ & $\begin{array}{c}\text { Fluence } \\
/\left(\text { ions } \cdot \mathrm{cm}^{2}\right) \\
\end{array}$ & $\begin{array}{c}\text { Electronic deposited } \\
\text { energy } /\left(\mathrm{keV} \cdot \mathrm{cm}^{3}\right)\end{array}$ & $\begin{array}{l}\text { Nuclear deposited } \\
\text { energy } /\left(\mathrm{keV} \cdot \mathrm{cm}^{3}\right)\end{array}$ & Dose/dpa \\
\hline \multirow{8}{*}{$\mathrm{Xe}^{26+}$} & \multirow{8}{*}{5} & \multirow{8}{*}{1.9} & $6.1 \times 10^{11}$ & $7.20 \times 10^{18}$ & $3.99 \times 10^{19}$ & $1 \times 10^{-3}$ \\
\hline & & & $1.8 \times 10^{12}$ & $2.12 \times 10^{19}$ & $1.18 \times 10^{20}$ & $3 \times 10^{-3}$ \\
\hline & & & $3.6 \times 10^{12}$ & $4.25 \times 10^{19}$ & $2.35 \times 10^{20}$ & $6 \times 10^{-3}$ \\
\hline & & & $6.1 \times 10^{12}$ & $7.20 \times 10^{19}$ & $3.99 \times 10^{20}$ & $1 \times 10^{-2}$ \\
\hline & & & $1.8 \times 10^{13}$ & $2.12 \times 10^{20}$ & $1.18 \times 10^{21}$ & $3 \times 10^{-2}$ \\
\hline & & & $6.1 \times 10^{13}$ & $7.20 \times 10^{20}$ & $3.99 \times 10^{21}$ & 0.1 \\
\hline & & & $3.0 \times 10^{14}$ & $3.54 \times 10^{21}$ & $1.96 \times 10^{22}$ & 0.5 \\
\hline & & & $6.1 \times 10^{14}$ & $7.20 \times 10^{21}$ & $3.99 \times 10^{22}$ & 1.0 \\
\hline \multirow{8}{*}{$\mathrm{Kr}^{17+}$} & \multirow{8}{*}{4} & \multirow{8}{*}{2.4} & $3.0 \times 10^{11}$ & $2.80 \times 10^{18}$ & $9.68 \times 10^{18}$ & $2.05 \times 10^{-4}$ \\
\hline & & & $1.0 \times 10^{12}$ & $9.34 \times 10^{18}$ & $3.23 \times 10^{19}$ & $6.83 \times 10^{-4}$ \\
\hline & & & $3.0 \times 10^{12}$ & $2.80 \times 10^{19}$ & $9.68 \times 10^{19}$ & $2.05 \times 10^{-3}$ \\
\hline & & & $6.0 \times 10^{12}$ & $5.61 \times 10^{19}$ & $1.94 \times 10^{20}$ & $4.10 \times 10^{-3}$ \\
\hline & & & $1.0 \times 10^{13}$ & $9.34 \times 10^{19}$ & $3.23 \times 10^{20}$ & $6.83 \times 10^{-3}$ \\
\hline & & & $3.0 \times 10^{13}$ & $2.80 \times 10^{20}$ & $9.68 \times 10^{20}$ & $2.05 \times 10^{-2}$ \\
\hline & & & $1.0 \times 10^{14}$ & $9.34 \times 10^{20}$ & $3.23 \times 10^{21}$ & $6.83 \times 10^{-2}$ \\
\hline & & & $5.0 \times 10^{14}$ & $4.67 \times 10^{21}$ & $1.61 \times 10^{22}$ & $3.41 \times 10^{-1}$ \\
\hline \multirow{8}{*}{$\mathrm{P}^{+}$} & \multirow{8}{*}{0.3} & \multirow{8}{*}{0.4} & $4.0 \times 10^{11}$ & $1.21 \times 10^{18}$ & $3.24 \times 10^{18}$ & $2.26 \times 10^{-4}$ \\
\hline & & & $1.4 \times 10^{12}$ & $4.25 \times 10^{18}$ & $1.14 \times 10^{19}$ & $7.95 \times 10^{-4}$ \\
\hline & & & $4.0 \times 10^{12}$ & $1.21 \times 10^{19}$ & $3.24 \times 10^{19}$ & $2.26 \times 10^{-3}$ \\
\hline & & & $8.0 \times 10^{12}$ & $2.43 \times 10^{19}$ & $6.49 \times 10^{19}$ & $4.52 \times 10^{-3}$ \\
\hline & & & $1.4 \times 10^{13}$ & $4.25 \times 10^{19}$ & $1.14 \times 10^{20}$ & $7.95 \times 10^{-3}$ \\
\hline & & & $4.0 \times 10^{13}$ & $1.21 \times 10^{20}$ & $3.24 \times 10^{20}$ & $2.26 \times 10^{-2}$ \\
\hline & & & $1.4 \times 10^{14}$ & $4.25 \times 10^{20}$ & $1.14 \times 10^{21}$ & $7.95 \times 10^{-2}$ \\
\hline & & & $7.0 \times 10^{14}$ & $2.13 \times 10^{21}$ & $5.68 \times 10^{21}$ & $3.94 \times 10^{-1}$ \\
\hline \multirow{6}{*}{$\mathrm{Au}$} & \multirow{6}{*}{8} & \multirow{6}{*}{2.0} & $1.14 \times 10^{12}$ & $2.14 \times 10^{19}$ & $1.39 \times 10^{20}$ & $2.65 \times 10^{-3}$ \\
\hline & & & $3.79 \times 10^{12}$ & $7.11 \times 10^{19}$ & $4.62 \times 10^{20}$ & $8.85 \times 10^{-3}$ \\
\hline & & & $7.57 \times 10^{12}$ & $1.42 \times 10^{20}$ & $9.23 \times 10^{20}$ & $1.77 \times 10^{-2}$ \\
\hline & & & $1.14 \times 10^{13}$ & $2.14 \times 10^{20}$ & $1.39 \times 10^{21}$ & $2.65 \times 10^{-2}$ \\
\hline & & & $3.79 \times 10^{13}$ & $7.11 \times 10^{20}$ & $4.62 \times 10^{21}$ & $8.85 \times 10^{-2}$ \\
\hline & & & $1.40 \times 10^{14}$ & $2.63 \times 10^{21}$ & $1.71 \times 10^{22}$ & $3.26 \times 10^{-1}$ \\
\hline
\end{tabular}

纳米压痕仪测量了辐照后样品的硬度和模量。基本 测量方式是用一定的力加载在金刚石压头上，金刚 石雉压头会在样品表面形成压痕。硬度是根据以下 公式计算得到的 ${ }^{[13]}$.

$$
H=\frac{P_{\max }}{A}
$$

其中 $H$ 是指材料的硬度, $P_{\text {max }}$ 是加载在金刚石上的 力, $A$ 是压痕的接触面积, 它是接触深度的函数。采 用连续刚度法测量, 即不断增加压头上的荷载, 同 时在荷载上附加上可调简谐荷载, 然后通过样品的 加载卸载曲线得到测试样品与压头的接触面积, 进 而得到样品的硬度和模量测量曲线 ${ }^{[14]}$ 。在实验中加载 纳米压痕仪压入深度为 $2 \mu \mathrm{m}$, 最大载荷为 $500 \mathrm{mN}$, 样品的泊松比为 0.22 。

模量是根据以下公式计算得到的:

$$
\begin{gathered}
E_{\mathrm{r}}=\frac{\sqrt{\pi}}{2} \cdot \frac{S}{\sqrt{A}} \\
\frac{1}{E_{\mathrm{r}}}=\frac{1-v^{2}}{E}+\frac{1-v_{i}^{2}}{E_{i}}
\end{gathered}
$$

其中 $E_{\mathrm{r}}$ 是等效弹性模量, $S$ 是刚度, $A$ 是压痕表面面 积, $E_{i} 、 v_{i}$ 分别为金刚石压头的弹性模量与泊松比, $E 、 v$ 分别为被测材料的弹性模量与泊松比。

为了探究辐照前后样品的结构的变化, 使用 $\mathrm{X}$ 射线衍射仪(荷兰帕纳科公司生产)对样品进行分析。 测试条件为 $\mathrm{Cu}$ 靶产生的波长是 $0.154 \mathrm{~nm}$ 的单色 $\mathrm{X}$ 射线, 采用掠入射(GIXRD)的方式对样品进行测量。 其入射角 $\alpha$ 为 $1^{\circ}$, 测试范围为 $2 \theta=10^{\circ} \sim 90^{\circ}$ 。

根据文献[15], 当 $X$ 射线以某个小角度入射到介 质表面时会发生全反射现象，这个临界角 $\alpha_{\mathrm{c}}$ 为: 


$$
\alpha_{\mathrm{c}}=\sqrt{2 \delta}=\sqrt{2.6 \times 10^{-6} \rho \lambda}
$$

式中 $\alpha_{c}$ 单位为弧度, $\rho$ 为材料密度 $\left(\mathrm{g} / \mathrm{cm}^{3}\right) ; \lambda$ 为 $X$ 射线 波长 $(0.154056 \mathrm{~nm})$ 。

当入射角 $\alpha_{i}<\alpha_{\mathrm{c}}$ 时, 对应的 X射线的穿透深度 $t$ 为:

$$
t=\frac{\lambda}{2 \pi\left(\alpha_{\mathrm{c}}^{2}-\alpha_{i}\right)^{1 / 2}}
$$

当入射角 $\alpha_{i}>\alpha_{\mathrm{c}}$ 时, $\mathrm{X}$ 射线的穿透深度 $t$ 与样品 的线吸收系数 $\mu$ 有关:

$$
t=\frac{\sin \alpha_{i}}{2 \mu}
$$

本工作的拉曼分析测试是在兰州大学物理科学 与技术学院的 Labram HR 800 (Horiba Jobin Yvon) 激光共聚焦拉曼光谱仪上进行的。测试工作使用的 是波长为 $532 \mathrm{~nm}$ 的激光, 显微聚焦系统的光栅刻度 为 $600 \mathrm{~g} / \mathrm{mm}$, 物镜的放大倍数为 UV X100。拉曼光 谱扫描的波数范围为 $200 \sim 1600 \mathrm{~cm}^{-1}$, 积分时间为 $60 \mathrm{~s} \times 3$ 次。激光到达样品表面的功率约为 $5 \mathrm{~mW}$, 因 此测试过程中激光加热对样品结构的影响可以忽略 不计。所有测试均在室温下进行。

\section{2 结果与讨论}

\section{1 实验结果}

\subsection{1 硬度与模量}

图 2 和图 3 为辐照前后硼硅酸盐玻璃的硬度和 模量。为了定量地描述玻璃样品辐照前后硬度和模 量的变化, $\mathrm{Kr} 、 \mathrm{Xe} 、 \mathrm{Au}$ 离子辐照后嗍硅酸盐玻璃的 硬度和模量绝对值是由 $300 \sim 800 \mathrm{~nm}$ 区域决定的, 而 $\mathrm{P}$ 离子的穿透深度大概只有 $400 \mathrm{~nm}$, 所以选取 100 200 nm 这个区间作为硼硅酸盐玻璃的硬度与 模量。根据 Peuget 等 ${ }^{[16]}$ 的研究, 给出压痕深度和测 量深度满足 2 倍关系, 300 800 nm 压痕深度对应的 实际测量深度是 600 1600 nm, 100 200 nm 压痕深度 对应的实际测量深度 200 400 nm。但是所有样品都 是从表面开始，一定会有表面的影响，所以 300 $800 \mathrm{~nm}$ 压痕深度实际上对应的是 $0 \sim 1600 \mathrm{~nm}$ 深度区 域内的硬度和模量, 100 200 nm 压痕深度实际上对 应的是 $0 \sim 400 \mathrm{~nm}$ 深度区域内的硬度和模量。对其 使用外推法 ${ }^{[17]}$ 进行数据处理, 从而得到辐照后的硼 硅酸盐玻璃的硬度和模量随辐照剂量的变化关系。

图 4、图 5 和图 6 给出了 $\mathrm{P} 、 \mathrm{Kr} 、 \mathrm{Xe} 、 \mathrm{Au}$ 离子 辐照条件下嗍硅酸盐玻璃硬度和模量随着剂量的变 化规律。 $X$ 轴为对应的辐照剂量, 单位为 $\mathrm{dpa}$ (平均 离位数每原子), 标志着材料损伤的程度。辐照剂量 与离子的核能量沉积的大小有关, 且正比于离子

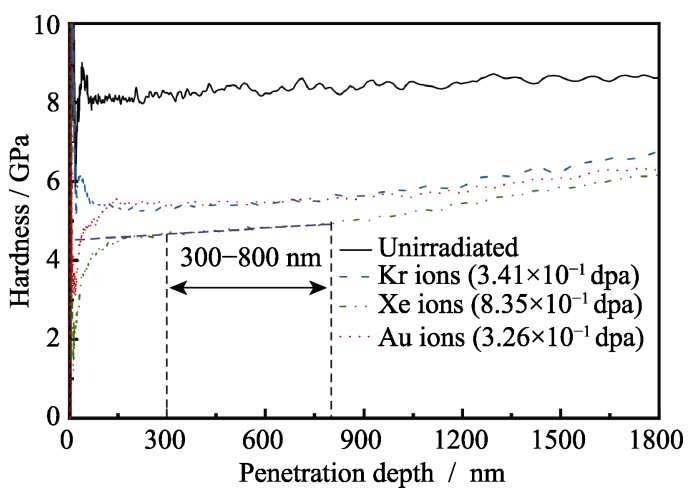

图 2 嗍硅酸盐玻璃的硬度随深度的变化曲线

Fig. 2 Hardness changing curves of borosilicate glass with penetration depth

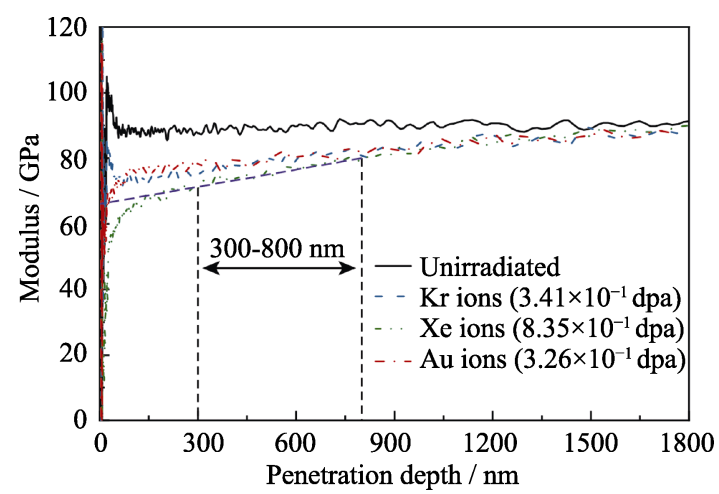

图 3 喼硅酸盐玻璃的模量随深度的变化曲线

Fig. 3 Modulus change of borosilicate glass with penetration depth

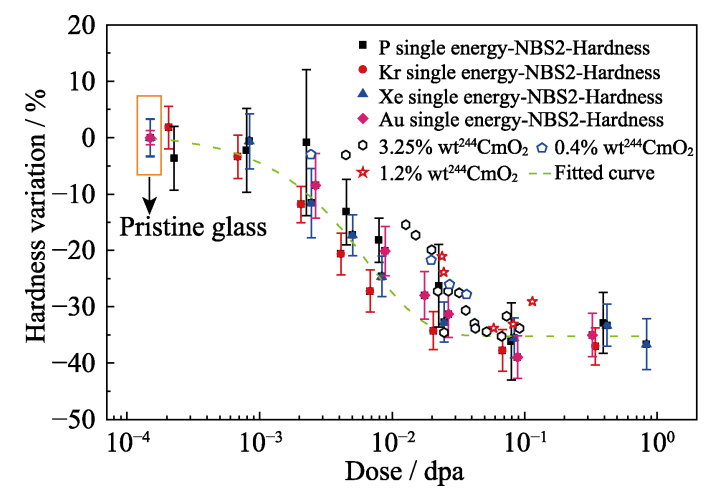

图 4 重离子辐照下嗍硅酸盐玻璃硬度随剂量的变化

Fig. 4 Hardness variation of borosilicate glass dose for the irradiation of heavy ions

与靶核弹性碰撞的传递能量。通过对比发现随着辐 照剂量的增大, $\mathrm{P} 、 \mathrm{Kr} 、 \mathrm{Xe} 、 \mathrm{Au}$ 离子辐照嗍硅酸盐玻 璃的硬度和模量都发生了显著的下降。当用离子辐 照样品时, 在剂量小于 $0.001 \mathrm{dpa}$ 时, 玻璃的硬度和模 量没有明显变化; 当辐照剂量达到 $0.001 \mathrm{dpa}$ 时, 玻璃 的硬度和模量开始迅速的下降, 分别为下降了 $3 \%$ 和 $1 \%$ 。当辐照剂量达到 $0.1 \mathrm{dpa}$ 时, 玻璃的硬度和模量 达到稳定值, 此时样品的硬度和模量分别下降了 $35 \%$ 和 $18 \%$ 。四种离子尽管种类和能量都不相同, 但 是辐照后玻璃的硬度和模量的变化趋势基本一致, 


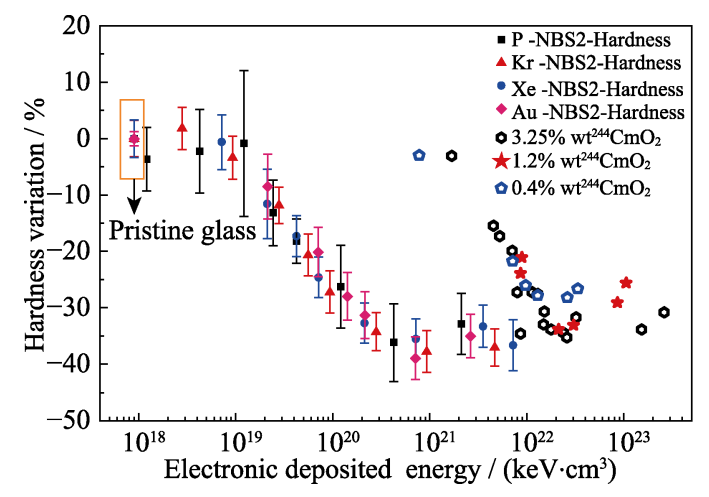

图 5 重离子辐照下嗍硅酸盐玻璃硬度随电子能量沉积的 变化

Fig. 5 Hardness variation of borosilicate glass with deposited electronic energy for the irradiation of heavy ions

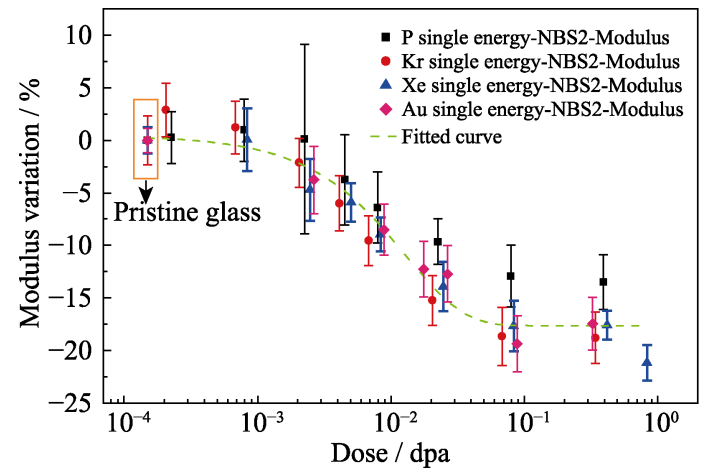

图 6 重离子辐照条件下嗍硅酸盐玻璃模量随剂量的变化

Fig. 6 Modulus variation of borosilicate glass with dose for the irradiation of heavy ions

都是先下降然后趋于稳定, 而且硬度最终的稳定值 相对于样品的初始值大致下降了 $35 \%$, 模量最终的 稳定值相对于样品的初始值大致下降了 $18 \%$ 。

重离子辐照后嗍硅酸盐玻璃样品硬度(模量)与 辐照剂量拟合满足如下关系:

$$
S=S_{\mathrm{P}}-\Delta S \times\left(1-e^{-H_{\mathrm{Dose}} / t}\right)
$$

其中: $S$ 为辐照后硼硅酸盐玻璃的硬度(模量)值, $S_{\mathrm{P}}$ 近似为未辐照硼硅酸盐玻璃硬度(模量)值, $\Delta S$ 近似 为未辐照硼硅酸盐玻璃硬度 (模量) 与辐照最大剂量 碀硅酸盐玻璃硬度(模量)之差, $H_{\text {Dose }}$ 为辐照剂量, $t$ 为衰减常数, 对应不同拟合曲线的 $t$ 是不相同的。表 2 给出了一些样品性能的拟合参数。

\subsubsection{GIXRD 分析}

图 7 给出了辐照前后嗍硅酸盐玻璃的 GIXRD 图谱。本次实验使用的嗍硅酸盐玻璃是经式(3)计算 得到临界角 $\alpha_{\mathrm{c}}=0.1739^{\circ}$, 而掠入射角 $\alpha_{i}=1^{\circ}$, 入射角 $\alpha_{i}>\alpha_{\mathrm{c}}$, 采用式(5)计算得到 $\mathrm{X}$ 射线穿透深度约为 $17 \mu \mathrm{m}, \mathrm{Kr} 、 \mathrm{Xe} 、 \mathrm{Au}$ 离子辐照层厚度约为 $2 \mu \mathrm{m}$, 因此 GIXRD 反映的是辐照层的结构信息。从图 7 中可以 看到未辐照玻璃的衍射峰为漫散衍射包, 是典型的 非晶结构。通过对比未辐照样品及经最大剂量重
表 2 玻璃样品性能的拟合参数

Table 2 Properties fitting of glass samples

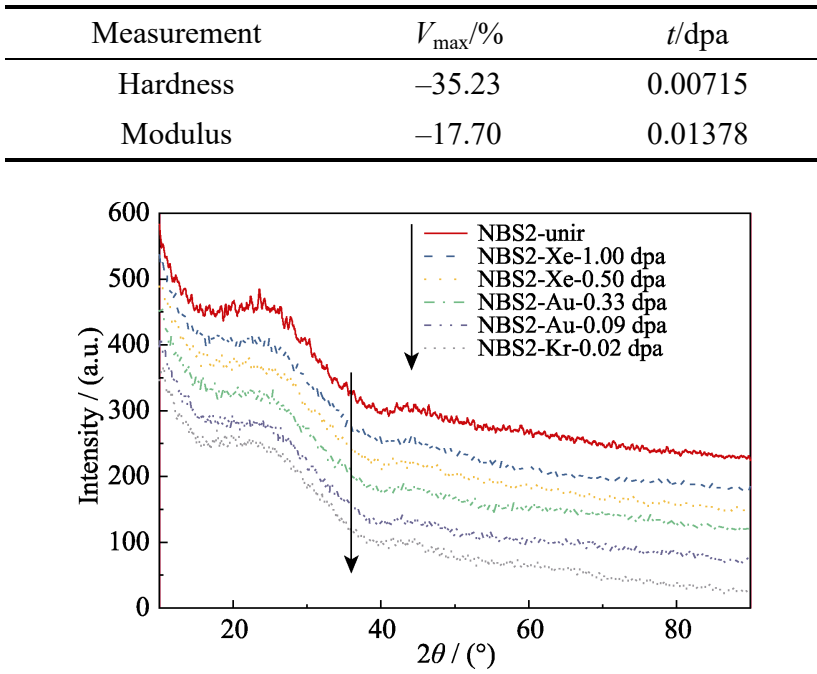

图 7 辐照前后嗍硅酸盐玻璃的 GIXRD 谱图

Fig. 7 GIXRD patterns of borosilicate glass pristine and irradiated with heavy ions

离子辐照后样品的 GIXRD 谱图, 可以发现辐照并未 使玻璃样品发生晶态结构的变化, 辐照前后样品均 为非晶态; 而且在 $\mathrm{Kr} 、 \mathrm{Xe} 、 \mathrm{Au}$ 三种不同离子和不 同剂量下辐照的样品的晶态结构并没有差异。

\subsection{3 拉曼光谱分析}

图 8 是 $\mathrm{Au}$ 离子辐照前后嗍硅酸盐玻璃的拉曼 谱图。位于 $500 \mathrm{~cm}^{-1}$ 左右的峰对应的是 $\mathrm{Si}-\mathrm{O}-\mathrm{Si}$ 单 元的弯曲振动模式 ${ }^{[5,18]}$, 随着辐照剂量的增大, 此 峰位从低波数迁移到高波数, 意味着 $\mathrm{Si}-\mathrm{O}-\mathrm{Si}$ 平均 键角减小 ${ }^{[5]}$ 。位于 $630 \mathrm{~cm}^{-1}$ 左右的峰对应的是类赛 黄晶环的呼吸振动模式 ${ }^{[19]}$ ，此处峰展宽，代表着类 赛黄晶环的解体。位于 $1060 \mathrm{~cm}^{-1}$ 左右的峰对应的 是 $\mathrm{Q}^{3}$ 结构单元 ${ }^{[18]}$, 它的变化会影响玻璃的聚合度。位 于 $1150 \mathrm{~cm}^{-1}$ 左右的峰对应的是 $Q^{4}$ 结构单元 ${ }^{[18]}$, 它 的变化也与玻璃的聚合度有关。但是由于辐照产生 的缺陷结构在拉曼测试时产生较强的荧光效应，它

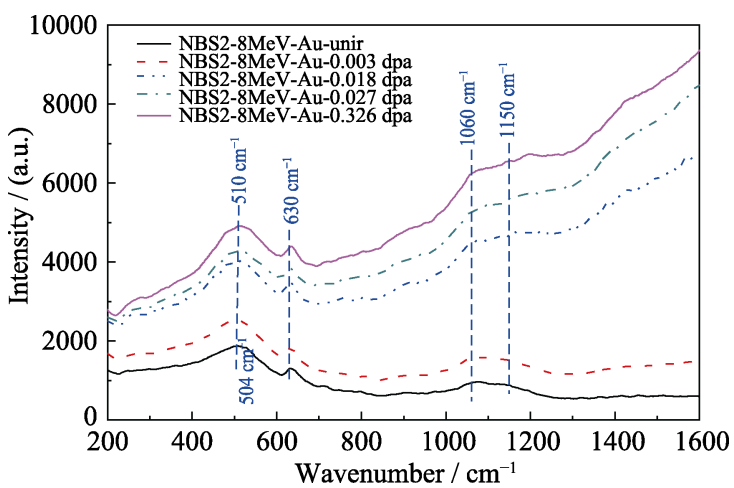

图 $8 \mathrm{Au}$ 离子辐照前后嗍硅酸盐玻璃的拉曼谱图

Fig. 8 Raman spectra of borosilicate glass pristine and irradiated with $\mathrm{Au}$ ions 
遮盖了一些样品本身的特征信息, 如在本次拉曼测 试中在辐照剂量超过 $0.003 \mathrm{dpa}$ 时, $1000 \sim 1600 \mathrm{~cm}^{-1}$ 处的苂光效应随着剂量的增大而增强, 所以无法从 拉曼谱的 1000 1600 $\mathrm{cm}^{-1}$ 处得到结构变化的信息。

\section{2 实验误差分析}

硬度和模量测量中的误差来源有两种: 统计误 差和系统误差。

$$
\delta_{\text {tot }}=\sqrt{\delta_{\text {sta }}^{2}+\delta_{\text {sys }}^{2}}
$$

其中 $\delta_{\mathrm{tot}}$ 是样品总相对误差, $\delta_{\mathrm{sta}}$ 由样品测量的统计 误差给出, $\delta_{\mathrm{sys}}$ 是系统误差。每个样品会选择五个点 测量硬度和模量, 其测量值的标准差就是统计误差, 测得硬度统计误差不超过 6\%, 模量统计误差不超 过 3\%。系统误差是在相同条件下测量熔融石英标 准样品的六个点的标准差, 测得硬度系统误差为 $3.3 \%$, 模量系统误差为 $1.5 \%$ 。硼硅酸盐玻璃样品硬 度总相对误差小于 $6.8 \%$, 模量总相对误差小于 $3.4 \%$ 。

\section{3 讨论}

图 4 和图 5 中为了说明硬度变化的规律, 引入 了 Peuget 等 ${ }^{[16]}$ 的结果作为对比。可以看出掺杂 ${ }^{244} \mathrm{Cm}$ 的玻璃硬度下降的结果与本工作中用辐照剂 量(dpa)表示的硬度变化的嗍硅酸盐玻璃的结果基 本一致。在 Peuget 等的研究中, 玻璃的硬度先下降 然后饱和, 最大硬度下降了大约 $30 \%$ 。而用电子能 损表示的硬度变化结果则出现了较大的差异, 本工 作中在电子能量沉积达到 $10^{19} \mathrm{keV} / \mathrm{cm}^{3}$ 时, 硬度开 始下降; 当电子能量沉积达到 $10^{20} \mathrm{keV} / \mathrm{cm}^{3}$ 时硬度 下降达到饱和, 此时硬度下降了 $35 \%$ 。而在 Peuget 等的工作中电子能量沉积达到 $10^{21} \mathrm{keV} / \mathrm{cm}^{3}$ 时, 硬 度才开始下降; 当电子能量沉积达到 $10^{22} \mathrm{keV} / \mathrm{cm}^{3}$ 时硬度下降达到饱和, 此时硬度下降了 $30 \%$ 。 Peuget 等对比了掺杂 ${ }^{244} \mathrm{Cm}$ 的玻璃硬度下降的结果, 指出导致玻璃硬度下降的原因主要是核能损而不是 电子能损。Peng 等 ${ }^{[20]}$ 也对比了离子辐照和 ${ }^{244} \mathrm{Cm}$ 掺 杂玻璃硬度下降的结果, 指出如果使用电子能量沉 积作为度量, 则离子辐照后的玻璃硬度下降将遵循 不同的规律; 而使用核能量沉积时, 玻璃硬度的下 降遵循相同的规律。

一般来说重离子辐照硼硅酸盐玻璃, 入射粒子 的能量损失分为两类: 电子能损和核能损。Karakurt 等 ${ }^{[21}$ 研究了 $\mathrm{He}$ 和 $\mathrm{Au}$ 离子对喼硅酸盐玻璃的辐照效 应, 他们发现对于 $\mathrm{He}$ 和 $\mathrm{Au}$ 离子辐照硼硅酸盐玻璃, 在核能量沉积相同时硬度下降程度基本相同, 但是 对应的电子能量沉积值却相差了约 150 倍, 他们的 结果表明主要是核能损导致玻璃硬度下降而不是电 子能损。最近 Mir 等 ${ }^{[22-23]}$ 分别用轻重粒子辐照 BS3
和 SON68 嗍硅酸盐玻璃, 指出核能损和电子能损都 可以造成玻璃硬度的下降, 但是只有当电子能损超 过 $4 \mathrm{keV} / \mathrm{nm}$, 电子才会对玻璃的硬度造成比较明显 的下降。但是本次实验所涉及的 $\mathrm{P} 、 \mathrm{Kr} 、 \mathrm{Xe} 、 \mathrm{Au}$ 离子能量分别为 $0.47 、 1.78 、 2.06 、 2.53 \mathrm{keV} / \mathrm{nm}$, 低 于这个间值。当电子能损低于这个间值时, 电子能 损主要导致靶原子激发电离, 很少造成靶原子离位, 无法破坏玻璃的网络体结构, 对玻璃硬度的影响较 小, 因此可以认为主要是核能损造成了辐照样品硬 度的下降。本次实验使用四种能量和种类不同的离 子辐照嗍硅酸盐玻璃, 但是四种离子造成的整体硬 度下降趋势基本一致, 低能量的离子和高能量的离 子对样品的损坏大致相同, 以后的辐照实验中可以 选择用低能量的离子来研究样品的辐照效应。

重离子辐照嗍硅酸盐玻璃时, 重离子会与靶核 发生弹性碰撞, 靶原子获得反冲能量而发生移位, 这会产生大量的缺陷结构, 如 $\mathrm{Si}-\mathrm{O}$ 键断裂, 导致玻 璃网络体结构发生改变, 无序度增大。辐照产生的 缺陷结构可能在拉曼测试时产生较强的苂光效应, 在拉曼测试中可以看到, 苂光效应随着辐照剂量的 增大而增强, 这也说明了随着辐照剂量的增大, 样 品中产生的缺陷增多。这对应于 $500 \mathrm{~cm}^{-1}$ 左右 $\mathrm{Si}-\mathrm{O}-\mathrm{Si}$ 平均键角的减小, 意味着玻璃无序度的增 强, 导致硬度下降 ${ }^{[24]}$ 。Chen 等 ${ }^{[25]}$ 通过研究利用 $\mathrm{Kr}$ 离子辐照同样观察到了 $\mathrm{Si}-\mathrm{O}-\mathrm{Si}$ 平均键角的减小, 通过拟合得到 $630 \mathrm{~cm}^{-1}$ 处的峰展宽, 对应的类赛黄 晶环的结构逐渐解体, 造成玻璃聚合度降低, 也同 样表明玻璃内部无序度增强。嗍结构单元的变化也 是导致玻璃硬度与模量下降的一个很重要的因素。 由于辐照产生的缺陷在拉曼测试中产生了很强的苂 光本底, 所以并未从拉曼谱中得到更多关于硼结构 的变化信息。但是 Yuan 等 ${ }^{[26]}$ 用分子动力学模拟了 $\mathrm{Xe}$ 辐照后玻璃微观结构的变化, 发现随着剂量的增 加, 硬度呈现先减小后趋于饱和的变化规律, 即硼 (B) 的配位数和硅氧嗍 $(\mathrm{Si}-\mathrm{O}-\mathrm{B})$ 的数量先减少后趋 于饱和, 混合网络结构 $(\mathrm{Si}-\mathrm{O}-\mathrm{B})$ 中的 $\mathrm{BO}_{4}$ 结构发生 断裂, 进而诱发了诸如 $\mathrm{B}$ 的配位数下降、 $\mathrm{BO}_{4}$ 转变 为 $\mathrm{BO}_{3}$ 和网络聚合度下降等现象, 进而导致了玻璃 硬度的下降。嗍氧四面体 $\mathrm{BO}_{4}$ 是三维空间架状, $\mathrm{BO}_{3}$ 是三角体结构单元, 即原来的三维空间网络结构会 转变成二维空间层状结构, 进而导致硬度和模量的 下降 ${ }^{[27]}$ 。Peng 等 ${ }^{[28]}$ 通过对比 $\mathrm{Xe}$ 辐照的石英玻璃和 硼硅酸盐玻璃硬度和模量的变化, 也认为玻璃中的 硼元素会对硬度与模量产生重要影响。玻璃微观结 构变化的外在表现就是样品的硬度还有模量等参数 的变化, 但是硬度与模量下降的具体机理还有待进 
一步的研究。

从 GIXRD 测试结果中可以得到在不同剂量并 且离子种类和能量都不相同的情况下, 辐照前后都 是一个与原始样品相同的非晶包, 具有典型的非晶 结构特性，辐照后硼硅酸盐玻璃仍保持非晶状态。 这主要是因为辐照过程中样品的温度始终低于玻璃 的晶化温度。当样品温度低于这个晶化温度时, 尽 管辐照剂量已经相当大 (最大剂量约为 $1 \mathrm{dpa})$, 样品 仍然保持非晶态结构。

\section{3 结论}

分别采用 $0.3 \mathrm{MeV}$ 的 $\mathrm{P}$ 离子、 $4 \mathrm{MeV}$ 的 $\mathrm{Kr}$ 离子、 $5 \mathrm{MeV}$ 的 $\mathrm{Xe}$ 离子以及 $8 \mathrm{MeV}$ 的 $\mathrm{Au}$ 离子辐照硼硅酸 盐玻璃, 利用纳米压痕技术表征了辐照前后样品的 硬度和模量的变化; 使用 GIXRD 对样品晶态结构进 行了分析; 利用 Raman 谱对辐照后样品的微观结构 进行了表征:

1) 重离子辐照后嗍硅酸盐玻璃硬度和模量明显 下降, 硬度与模量呈现出先减小后趋于饱和的变化 规律, 四种离子造成的硬度和模量下降趋势基本一 致, 低能量和高能量的离子对样品的损坏大致相同, 喼硅酸盐玻璃样品硬度下降 35\%, 模量下降 18\%;

2) 硬度和模量下降主要是由核能损导致的。此 外, 辐照会使玻璃网络结构发生改变, 玻璃的聚合 度下降, 无序度增强;

3) 辐照采用的离子种类、能量以及辐照剂量不 会对样品的晶态结构产生影响, 辐照后嗍硅酸盐玻 璃仍保持非晶状态;

4) 本研究发现低能量和高能量的离子对样品的 损坏大致相同。

\section{参考文献:}

[1] WEBER W J, EWING R C, ANGELL C A, et al. Radiation effects in glasses used for immobilization of high-level waste and plutonium disposition. Journal of Materials Research, 1997, 12(8): 1948-1978.

[2] JANTZEN C, KAPLAN D, BIBLER N, et al. Performance of a buried radioactive high level waste (HLW) glass after 24 years. Journal of Nuclear Materials, 2008, 378(3): 244-256.

[3] WADE B. The management of radioactive wastes. Physics in Technology, 1982, 13(3): 112.

[4] PEUGET S, FARES T, MAUGERI E A, et al. Effect of 10 B(n, $\alpha)$ $7 \mathrm{Li}$ irradiation on the structure of a sodium borosilicate glass. Nuclear Instruments \& Methods in Physics Research, 2014, 327(1): 22-28.

[5] BONFILS J D, PEUGET S, PANCZER G, et al. Effect of chemical composition on borosilicate glass behavior under irradiation. Journal of Non-Crystalline Solids, 2010, 356(6): 388-393.

[6] WEBER W J. Models and mechanisms of irradiation-induced amorphization in ceramics. Nuclear Instruments \& Methods in Physics Research, 2000, 166-167(99): 98-106.

[7] ABBAS A, SERRUYS Y, GHALEB D, et al. Evolution of nuclear glass structure under $\alpha$-irradiation. Nuclear Instruments \& Methods in Physics Research, 2000, 166-167(166): 445-450.

[8] OLLIER N, BOIZOT B, REYNARD B, et al. Beta irradiation borosilicate glasses: the role of the mixed alkali effect. Nuclear Inst \& Methods in Physics Research B, 2006, 218(1): 176-182.

[9] ZHANG G F, WANG T S, YANG K J, et al. Raman spectra and nano-indentation of Ar-irradiated borosilicate glass. Nuclear Instruments \& Methods in Physics Research, 2013, 316(12): 218-221.

[10] YANG K J, WANG T S, ZHANG G F, et al. Study of irradiation damage in borosilicate glass induced by $\mathrm{He}$ ions and electrons. Nuclear Inst \& Methods in Physics Research B, 2013, 307(6): 541-544.

[11] LUO S, LI L, TANG B, et al. Synroc immobilization of high level waste (HLW) bearing a high content of sodium. Waste Management, 1998, 18(1): 55-59.

[12] DELAYE J M, PEUGET S, BUREAU G, et al. Molecular dynamics simulation of radiation damage in glasses. Journal of Non-Crystalline Solids, 2011, 357(14): 2763-2768.

[13] JOSLIN D L, OLIVER W C. A new method for analyzing data from continuous depth-sensing microindentation tests. Journal of Materials Research, 1990, 5(1): 123-126.

[14] ROUTBORT J L, MATZKE H. The effect of composition and radiation on the fracture of a nuclear waste glass. Materials Science \& Engineering, 1983, 58(2): 229-237.

[15] 莫志深, 张宏放. 晶态聚合物结构和 X射线衍射. 北京: 科学出 版社, 2003: 360-361

[16] PEUGET S, NOËL P Y, LOUBET J L, et al. Effects of deposited nuclear and electronic energy on the hardness of R7T7-type containment glass. Nuclear Instruments \& Methods in Physics Research, 2006, 246(2): 379-386.

[17] PENG H, SUN M, LIU F, et al. Potential effect on the interaction of highly charged ion with graphene. Nuclear Instruments \& Methods in Physics Research, 2017, 407: 291-296.

[18] MANARA D, GRANDJEAN A, NEUVILLE D. Advances in understanding the structure of borosilicate glasses: a Raman spectroscopy study. American Mineralogist, 2009, 94(5/6): 777-784.

[19] BOIZOT B, AGNELLO S, REYNARD B, et al. Raman spectroscopy study of $\beta$-irradiated silica glass. Journal of NonCrystalline Solids, 2003, 325(1/2/3): 22-28.

[20] PENG H B, SUN M L, YANG K J, et al. Effect of irradiation on hardness of borosilicate glass. Journal of Non-Crystalline Solids, 2016, 443: 143-147.

[21] KARAKURT G, ABDELOUAS A, GUIN J P, et al. Understanding of the mechanical and structural changes induced by alpha particles and heavy ions in the French simulated nuclear waste glass. Journal of Nuclear Materials, 2016, 475: 243-254.

[22] MIR A H, MONNET I, TOULEMONDE M, et al. Mono and sequential ion irradiation induced damage formation and damage recovery in oxide glasses: stopping power dependence of the mechanical properties. Journal of Nuclear Materials, 2016, 469: 244-250.

[23] MIR A H, TOULEMONDE M, JEGOU C, et al. Understanding and simulating the material behavior during multi-particle irradiations. Scientific Reports, 2016, 6: 30191-1-11.

[24] KIEU L H, KILYMIS D, DELAYE J M, et al. Discussion on the structural origins of the fracture toughness and hardness changes in rapidly quenched borosilicate glasses: a molecular dynamics study. Procedia Materials Science, 2014, 7: 262-271.

[25] CHEN L, WANG T S, ZHANG G F, et al. XPS and Raman studies of electron irradiated sodium silicate glass. Chinese Physics $B$, 2013, 22(12): 126101-1-6.

[26] YUAN W, PENG H, SUN M, et al. Structural origin of hardness decrease in irradiated sodium borosilicate glass. Journal of Chemical Physics, 2017, 147(23): 234502-1-6.

[27] KILYMIS D A, DELAYE J M. Nanoindentation studies of simplified nuclear glasses using molecular dynamics. Journal of Non-Crystalline Solids, 2014, 401(1430): 147-153.

[28] PENG H B, LIU F F, ZHANG B T, et al. Comparative studies of irradiation effects in borosilicate glass and fused silica irradiated by energetic Xe ions. Acta Physica Sinica, 2018, 67(3): 038101$1-8$. 